\title{
ANTIBACTERIAL ACTIVITY OF MEDICINAL PLANT EXTRACTS
}

\author{
Priscila Ikeda Ushimaru ${ }^{1}$; Mariama Tomaz Nogueira da Silva ${ }^{1}$; Luiz Claudio Di Stasi ${ }^{2}$; Luciano Barbosa ${ }^{3}$; \\ Ary Fernandes Junior ${ }^{1 *}$
}

${ }^{1}$ Departamento de Microbiologia e Imunologia, Instituto de Biociências de Botucatu, Universidade Estadual Paulista, Botucatu, SP, Brasil; ${ }^{2}$ Departamento de Farmacologia, Instituto de Biociências de Botucatu, Universidade Estadual Paulista, Botucatu, SP, Brasil; ${ }^{3}$ Departamento de Bioestatística, Instituto de Biociências de Botucatu, Universidade Estadual Paulista, Botucatu, SP, Brasil

Submitted: February 14, 2007; Returned to authors for corrections: May 19, 2007; Approved: September 27, 2007.

\section{SHORT COMMUNICATION}

\begin{abstract}
The present study aimed at evaluating the in vitro antimicrobial activity of methanolic extracts of some medicinal plants against Escherichia coli, Salmonella Typhimurium, Staphylococcus aureus and Enterococcus sp. The methanolic extract of Caryophyllus aromaticus presented the highest anti-S. aureus activity and was effective against all bacterial strains tested.
\end{abstract}

Key words: medicinal plants, antibacterial activity, Minimal Inhibitory Concentration

Further acquaintance with different ethnic groups has contributed to the development of research on natural products, to the increase in knowledge about the close relationship between the chemical structure of a certain compound and its biological properties, and to the understanding of the animal/ insect-plant interrelation (8). For these reasons, medicinal plants are important substances for the study of their traditional uses through the verification of pharmacological effects and can be natural composite sources that act as new anti-infectious agents.

The present study aimed at evaluating the in vitro antimicrobial activity of plant (Allium sativum, Zingiber officinale, Caryophyllus aromaticus, Cymbopogon citratus, Mikania glomerata and Psidium guajava) extracts against Gram-positive and Gram-negative bacterial strains isolated from human infections.

For the preparation of plant extracts, samples of A. sativum (bulbs), Z. officinale (rhizomes) and C. aromaticus (flower buds) were obtained at the local commerce in April 2006 and used in natura. Cymbopogon citratus (leaves) and Psidium guajava (leaves) were collected in May 2006 from an experimental field of the School of Agronomical Sciences, Unesp, Botucatu, São Paulo, Brazil. Mikania glomerata (leaves) was collected around
Demétria farm, Botucatu, São Paulo, during the same period. The leaves were dried at approximately $50^{\circ} \mathrm{C}$ and triturated in a mechanical mill. The voucher specimens were deposited in the Herbarium of the Department of Botany, Institute of Biosciences, Unesp, Botucatu-SP.

The determined plant parts $(200 \mathrm{~g})$ were ground, extracted with $70 \%$ methanol and filtered after $48 \mathrm{hs}$. The plant residue was re-extracted by adding $70 \%$ methanol and filtered again after $48 \mathrm{hs}$. Such procedure was repeated every $72 \mathrm{hs}$, completing three filtration processes. The filtrate was concentrated on a rotary evaporator at $45^{\circ} \mathrm{C}$ for methanol elimination, and the extracts were kept in sterile bottles under refrigerated conditions until use. The dry weight of the extracts was obtained by allowing the solvent to evaporate and was used to determine concentration in $\mathrm{mg} / \mathrm{mL}$. (Methodology based on Betoni et al. (3); Table 1).

Microbial susceptibility assays using the agar dilution (Mueller-Hinton Agar) method (\%v/v and corresponding mg/ $\mathrm{mL}$ values) and the Minimal Inhibitory Concentration (MIC) were carried out for fifteen Salmonella Typhimurium, S. aureus, Enterococcus sp and E. coli strains plus one ATCC strain of each bacterium. Overnight cultures $\left(37^{\circ} \mathrm{C}\right)$ in Brain Heart

*Corresponding Author. Mailing address: Departamento de Microbiologia e Imunologia, IBB/UNESP, Botucatu, SP, Brasil. Tel.: (14) 3811-6058 FAX (14) 3815-3744. E-mail: ary@ibb.unesp.br 
Table 1. Characteristics of the plants extracts.

\begin{tabular}{lccc}
\hline Scientific name & Common name & Part of the plant used & Extract dry weight (mg/mL) \\
\hline Allium sativum & Garlic & Bulbs & 133.0 \\
Caryophyllus aromaticus & Clove & Flower buds & 95.0 \\
Zingiber officinale & Ginger & Rhizomes & 17.0 \\
Psidium guajava & Guava & Leaves & 122.0 \\
Cymbopogon citratus & Lemongrass & Leaves & 75.0 \\
Mikania glomerata & "Guaco" & Leaves & 70.0 \\
\hline
\end{tabular}

Infusion (BHI) were adjusted to 0.5 Mac Farland standard and inoculated on Petri plates by using a Steer's replicator. After $37^{\circ} \mathrm{C} / 24$ hours, MIC values $(4,6)$ were read and MIC $50 \%$ and $90 \%$ values calculated. Kruskal-Wallis test for significant analysis $(\mathrm{p}<0.05)$ and Dunn's Test for multiple comparisons were carried out. Then, the results mean values, which represent the inhibitory capacity of each plant extract against the bacteria tested, were obtained and expressed as \%v/v and $\mathrm{mg} / \mathrm{mL}$ (Table 2).
MIC 90\% values were different among extracts. Garlic and ginger extracts showed high antimicrobial action against Gramnegative strains. Gram-positive bacteria were more susceptible to the other plant extracts. Clove extracts were highly effective against bacterial strains, especially $S$. aureus strain (Table 2).

The antimicrobial properties of medicinal plants has been explained by the chemical association of active substances; however, the activity of their extracts is not related to their respective dry weights, which can be proven when more

Table 2. Minimal inhibitory concentrations (MIC 50\% and 90\%) and mean values (\%v/v and mg/mL) according to the plant extracts against Escherichia coli, Salmonella, Staphylococcus aureus and Enterococcus sp.

\begin{tabular}{|c|c|c|c|c|c|c|c|c|c|}
\hline \multirow[t]{2}{*}{$\begin{array}{c}\text { Plant } \\
\text { Extracts }\end{array}$} & \multirow[t]{2}{*}{$\begin{array}{c}\text { Bacteria } \\
\text { spp. }\end{array}$} & \multicolumn{2}{|c|}{$\begin{array}{c}\text { Escherichia } \\
\text { coli }\end{array}$} & \multicolumn{2}{|c|}{ Salmonella } & \multicolumn{2}{|c|}{$\begin{array}{c}\text { Staphylococcus } \\
\text { aureus }\end{array}$} & \multicolumn{2}{|c|}{$\begin{array}{c}\text { Enterococcus } \\
\text { sp }\end{array}$} \\
\hline & & $\% \mathrm{v} / \mathrm{v}$ & $\mathrm{mg} / \mathrm{mL}$ & $\% \mathrm{v} / \mathrm{v}$ & $\mathrm{mg} / \mathrm{mL}$ & $\% \mathrm{v} / \mathrm{v}$ & $\mathrm{mg} / \mathrm{mL}$ & $\% \mathrm{v} / \mathrm{v}$ & $\mathrm{mg} / \mathrm{mL}$ \\
\hline \multirow[t]{3}{*}{ Garlic } & MIC50\% & 1.00 & 1.33 & 1.06 & 1.41 & 2.00 & 2.66 & 3.25 & 4.32 \\
\hline & MIC90\% & 1.04 & 1.38 & 1.21 & 1.61 & 2.00 & 2.66 & 3.67 & 4.88 \\
\hline & Median & $1.000 \mathrm{a}$ & $1.330 \mathrm{a}$ & $1.100 \mathrm{a}$ & $1.460 \mathrm{a}$ & $2.000 \mathrm{~b}$ & $2.660 \mathrm{~b}$ & $3.375 \mathrm{a}$ & $4.485 \mathrm{~b}$ \\
\hline \multirow[t]{3}{*}{ Clove } & MIC50\% & 1.22 & 1.16 & 1.60 & 1.52 & 0.41 & 0.39 & 1.15 & 1.09 \\
\hline & МIC90\% & 1.68 & 1.60 & 1.76 & 1.67 & 0.49 & 0.46 & 1.31 & 1.24 \\
\hline & Median & $1.800 \mathrm{a}$ & $1.710 \mathrm{a}$ & $1.800 \mathrm{a}$ & $1.710 \mathrm{a}$ & $0.500 \mathrm{a}$ & $0.470 \mathrm{a}$ & $1.350 \mathrm{a}$ & $1.280 \mathrm{a}$ \\
\hline \multirow[t]{3}{*}{ Guava } & MIC50\% & $3 ., 70$ & 4.51 & 1.90 & 2.32 & 0.55 & 0.67 & 1.25 & 1.52 \\
\hline & МIC90\% & 7.40 & 9.03 & 2.18 & 2.66 & 0.63 & 0.77 & 1.43 & 1.74 \\
\hline & Median & $4.000 \mathrm{a}$ & $4.880 \mathrm{~b}$ & $2.250 \mathrm{a}$ & $2.740 \mathrm{a}$ & $0.650 \mathrm{a}$ & $0.790 \mathrm{a}$ & $1.250 \mathrm{a}$ & $1.520 \mathrm{a}$ \\
\hline \multirow[t]{3}{*}{ Guaco } & MIC50\% & 30.00 & 21.00 & 30.00 & 21.00 & 4.67 & 3.27 & 12.86 & 9.00 \\
\hline & МIC90\% & 32.33 & 22.63 & 30.00 & 21.00 & 6.20 & 4.34 & 15.14 & 10.60 \\
\hline & Median & $30.000 \mathrm{~b}$ & $21.000 \mathrm{c}$ & $30.000 \mathrm{~b}$ & $21.000 \mathrm{c}$ & $5.000 \mathrm{~b}$ & $3.500 \mathrm{~b}, \mathrm{c}$ & $15.000 \mathrm{~b}$ & $10.500 \mathrm{c}, \mathrm{d}$ \\
\hline \multirow[t]{3}{*}{ Lemongrass } & MIC50\% & 40.00 & 30.00 & 40.37 & 30.28 & 19.00 & 14.25 & 17.50 & 13.12 \\
\hline & MIC90\% & 40.80 & 30.60 & 40.87 & 30.65 & 21.80 & 16.35 & 21.20 & 15.90 \\
\hline & Median & $40.500 \mathrm{~b}$ & $30.375 \mathrm{c}$ & $41.000 \mathrm{~b}$ & $30.750 \mathrm{c}$ & $19.500 \mathrm{c}$ & $14.625 \mathrm{~d}$ & $18.250 \mathrm{~b}$ & $13.685 \mathrm{~d}$ \\
\hline \multirow[t]{3}{*}{ Ginger } & MIC50\% & 41.00 & 6.97 & 41.00 & 6.97 & 45.11 & 7.67 & 46.00 & 7.82 \\
\hline & MIC90\% & 41.00 & 6.97 & 41.00 & 6.97 & 45.82 & 7.79 & 46.00 & 7.82 \\
\hline & Median & $41.000 \mathrm{~b}$ & $6.970 \mathrm{~b}$ & $41.000 \mathrm{~b}$ & $6.970 \mathrm{~b}$ & $46.000 \mathrm{~d}$ & $7.820 \mathrm{c}$ & $46.000 \mathrm{c}$ & $7.820 \mathrm{~b}, \mathrm{c}$ \\
\hline
\end{tabular}

Letters a, b, c and d: Means in the same column not followed by the same letter are significantly different $(p<0.001)$. 
effective extracts are considered, e.g. clove extract $(95 \mathrm{mg} / \mathrm{mL})$ which had relatively lower activity than guava $(122 \mathrm{mg} / \mathrm{mL})$ and garlic $(133 \mathrm{mg} / \mathrm{mL})$ extracts, and all these extracts showed similar antimicrobial activity patterns. Such results were different from data reported in literature. Samy (7) used methanolic extracts of ginger which did not present antimicrobial effect against $S$. aureus and E. coli. However, Indu et al. (5), using a different method of ginger extract preparation, verified an inhibitory action against $E$. coli as well as high antimicrobial activity of garlic extracts against E. coli and Salmonella.

Ahmad and Aqil (2) concluded that ethanolic extracts of garlic did not have anti-E. coli or anti-Shigella action. Using another methodology, Vuddhakul et al. (9) observed that garlic extracts inhibited the growth of V. parahaemolyticus, E. coli and $S$. aureus; however, lemongrass and ginger extracts did not show any antimicrobial activity. Such behavior of the antibacterial action was also verified by Adonizio et al. (1), who used lemongrass extracts and did not observe antibacterial effects.

Comparisons with pertinent data from literature indicate that, according to the methodology adopted in studies on antimicrobial activity, the most diverse results can be obtained. Plant extracts have shown inhibitory effect on the growth of the bacteria studied, although of distinct forms. It is therefore recommended that the nature and the number of the active antibacterial principles involved in each plant extract be studied in detail.

\section{ACKNOWLEDGMENTS}

This work was supported by Fundação de Amparo a Pesquisa do Estado de São Paulo (FAPESP, Brazil). The authors thank Dr. Lin Chau Ming (FCA/Unesp/Botucatu) for providing the plant specimens and Dr. Maria de Lourdes Ribeiro de Souza da Cunha (IBB/Unesp/Botucatu) for supplying S. aureus strains.

\section{RESUMO}

\section{Atividade antibacteriana de extratos de plantas medicinais}

Avaliou-se a atividade antimicrobiana in vitro de extratos metanólicos de algumas plantas medicinais frente a Escherichia coli, Salmonella Typhimurium, Staphylococcus aureus e Enterococcus sp. O extrato metanólico de Caryophyllus aromaticus foi o mais eficaz para todas as bactérias testadas $\mathrm{e}$ apresentou a melhor atividade anti-S. aureus.

Palavras-chave: plantas medicinais, atividade antibacteriana, concentração inibitória mínima

\section{REFERENCES}

1. Adonizio, A.L.; Downum, K.; Bennett, B.C.; Mathee, K. (2006). Anti-quorum sensing activity of medicinal plants in southern Florida. J. Ethnopharmacol., 105: 427-435.

2. Ahmad, I.; Aqil, F. (2007). In vitro efficacy of bioactive extracts of 15 medicinal plants against ES $\beta$ L-producing multidrug-resistant enteric bacteria. Microbiol. Res., 162: 264-275.

3. Betoni, J.E.C.; Mantovani, R.P.P.; Barbosa, L.N.; Di Stasi, L.C.; Fernandes Junior, A. (2006). Synergism between plant extract and antimicrobial drugs used on Staphylococcus aureus diseases. Mem. Inst. Oswaldo Cruz., 101: 387-390.

4. Clinical and Laboratory Standards Institute (CLSI). (2005). Performance standards for antimicrobial susceptibility testing. Fifteenth informational supplement. CLSI document M100-S15. Wayne, PA, USA.

5. Indu, M.N.; Hatha, A.A.M.; Abirosh, C.; Harsha, U.; Vivekanandan, G. (2006). Antimicrobial activity of some of the south-Indian spices against serotypes of Escherichia coli, Salmonella, Listeria monocytogenes and Aeromonas hydrophila. Braz. J. Microbiol. 37: 153-158.

6. National Commitee for Clinical Laboratory Standards (NCCLS). (2003). Methods for dilution antimicrobial susceptibility tests for bacteria that grow aerobically; Approved Standard - Sixth Edition NCCLS document M7-A6. Wayne, PA, USA.

7. Samy, R.P. (2005). Antimicrobial activity of some medicinal plants from India. Fitoterapia., 76: 697-699.

8. Viegas, C.; Bolzani, V.S. (2006). Os produtos naturais e a química medicinal moderna. Quím. Nova, 29: 326-337.

9. Vuddhakul, V.; Bhooponga, P.; Hayeebilana, F.; Subhadhirasakulb, S. (2007). Inhibitory activity of Thai condiments on pandemic strain of Vibrio parahaemolyticus. Food Microbiol., 24: 413-418. 